\title{
RISCO DE QUEDA DE PACIENTES HOSPITALIZADOS: FATORES DE RISCO E ATUAÇÕES DE ENFERMAGEM
}

\section{Risk of fall in hospitalized patients: risk factors and nursing interventions \\ Riesgo de caída en pacientes ingresados: factores de riesgo e intervenciones de Enfermería}

\author{
Maria Aline Moreira Ximenes iD \\ Universidade Estadual Vale do Acaraú - UVA - Sobral (CE) - Brasil \\ Jefferson Ribeiro Aguiar iD \\ Universidade Estadual Vale do Acaraú - UVA - Sobral (CE) - Brasil \\ Ismael Brioso Bastos (iD \\ Universidade Estadual do Ceará - UECE - Fortaleza (CE) - Brasil \\ Liliane Vieira de Sousa (D) \\ Universidade Estadual Vale do Acaraú - UVA - Sobral (CE) - Brasil \\ Joselany Áfio Caetano iD \\ Universidade Federal do Ceará - UFC - Fortaleza (CE) - Brasil \\ Lívia Moreira Barros \\ Universidade Estadual Vale do Acaraú - UVA - Sobral (CE) - Brasil
}

\section{RESUMO}

Objetivo: Identificar fatores de risco de queda em pacientes hospitalizados e propor atuações de Enfermagem. Métodos: Estudo avaliativo, descritivo e quantitativo, desenvolvido de junho a setembro de 2017, realizado em entidade filantrópica localizada em Sobral, Ceará, Brasil. A coleta incluiu 155 participantes, sendo realizada a partir de instrumento estruturado contendo fatores de risco de queda, conforme taxonomia da North American Nursing Diagnosis Association (NANDA-I). Utilizou-se a análise descritiva com frequências absoluta e relativa. Resultados: A amostra de 155 participantes evidenciou que $74,83 \%(n=116)$ eram homens, $54,83 \%(n=85)$ trabalhadores ativos, $62,58 \%(n=97)$ eram casados, $84,45 \%(n=134)$ católicos e $68,38 \%(n=106)$ tinham ensino fundamental completo. Os principais fatores de risco identificados foram: dificuldade na marcha $(56,77 \% ; n=88)$, mobilidade física prejudicada $(50,96 \% ; n=79)$, equilíbrio prejudicado $(47,74 \% ; n=74)$. Também esteve presente o histórico de quedas e o uso de dispositivos auxiliares, ambos com frequência de $27,74 \%(n=43)$. Quanto ao ambiente, o principal fator de risco foi material antiderrapante insuficiente no banheiro, com $26,67 \%(n=46)$. Com base nesses resultados, propuseram-se ações contemplando as dimensões cognitivas e ambientais, o equilíbrio, a transferência, o uso de dispositivos de saúde, a educação em saúde e as estruturas hospitalares. Conclusão: Dificuldade na marcha, mobilidade física prejudicada, equilíbrio prejudicado e uso de dispositivos auxiliares são os principais fatores de risco mapeados. A identificação do risco de quedas e a prescrição de atuações de Enfermagem adequadas podem favorecer a prevenção da ocorrência desse evento adverso no hospital avaliado.

Descritores: Acidentes por Quedas; Diagnóstico de Enfermagem; Processo de Enfermagem; Classificação; Cuidados de Enfermagem.

\section{ABSTRACT}

Objective: To identify risk factors for falls in hospitalized patients and propose nursing actions. Methods: This is an evaluative, descriptive and quantitative study, developed from June to September 2017, carried out in a philanthropic entity located in Sobral, Ceará, Brazil. The collection included 155 participants, being carried out using a structured instrument containing risk factors for falls, according to the taxonomy of the North American Nursing Diagnosis Association (NANDA-I). Descriptive analysis with absolute and relative frequencies was used. Results: The sample of 155 participants showed that $74.83 \%(n=116)$ were men, $54.83 \%(n=85)$ active workers, $62.58 \%(n=97)$ were married, $84.45 \%(n=134)$ Catholics and $68.38 \%(n=106)$ had completed

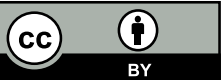


elementary school. The main risk factors identified were: difficulty in walking (56.77\%; $n=88)$, impaired physical mobility (50.96\%; $n=79)$, and impaired balance (47.74\%; $n=74)$. There was also a history of falls and the use of auxiliary devices, both with a frequency of $27.74 \%(n=43)$. As for the environment, the main risk factor was insufficient non-slip material in the bathroom, with $26.67 \%(n=46)$. Based on these results, actions were proposed covering the cognitive and environmental dimensions, balance, transfer, the use of health devices, health education, and hospital structures. Conclusion: Difficulty in walking, impaired physical mobility, impaired balance, and use of auxiliary devices are the main risk factors mapped. The identification of the risk of falls and the prescription of appropriate nursing actions can favor the prevention of the occurrence of this adverse event in the evaluated hospital.

Descriptors: Accidental Falls; Nursing Diagnosis; Nursing Process; Classification; Nursing Care.

\section{RESUMEN}

Objetivo: Identificar los factores de riesgo de caídas en pacientes ingresados y proponer actuaciones de Enfermería. Métodos: Estudio evaluativo, descriptivo y cuantitativo desarrollado entre junio y septiembre de 2017 realizado en una entidad filantrópica de Sobral, Ceará, Brasil. La recogida de datos incluyó 155 participantes y ha sido realizada a partir de un instrumento estructurado con los factores de riesgo de caídas según la taxonomía de la North American Nursing Diagnosis Association (NANDA-I). Se utilizó el análisis descriptivo con frecuencias absoluta y relativa. Resultados: La muestra de 155 participantes ha evidenciado que el $74,83 \%(n=116)$ eran hombres, el 54,83\% ( $n=85)$ eran trabajadores activos, el 62,58\% (n=97) eran casados, el $84,45 \%(n=134)$ eran católicos y el 68,38\% ( $n=106)$ tenían la educación primaria completa. Los principales factores de riesgo identificados fueron: la dificultad de la marcha (56,77\%; $n=88)$, la movilidad física perjudicada $(50,96 \% ; n=79)$ y el equilibrio perjudicado $(47,74 \% ; n=74)$. Se ha identificado también el histórico de caídas y el uso de dispositivos auxiliares ambos con una frecuencia del $27,74 \%$ ( $n=43$ ). Sobre el ambiente, el principal factor de riesgo ha sido el material antideslizante insuficiente en el baño en el 26,67\% ( $n=46)$ de los casos. Con base en eses resultados, se han propuesto acciones que incluyen las dimensiones cognitivas y ambientales, el equilibrio, la transferencia, el uso de dispositivos de salud, la educación en salud y las estructuras hospitalarias. Conclusión: La dificultad de la marcha, la movilidad física perjudicada, el equilibrio perjudicado y el uso de dispositivos auxiliares son los principales factores de riesgo presentados en el estudio. La identificación del riesgo de caídas y la prescripción de actuaciones de Enfermería adecuadas pueden favorecer la prevención de ese evento adverso en el hospital evaluado.

Descriptores: Accidentes por Caídas; Diagnóstico de Enfermería; Proceso de Enfermería; Clasificación; Atención de Enfermería.

\section{INTRODUÇÃO}

A queda, um evento que faz com que o indivíduo descanse inadvertidamente no solo, no chão ou em outro nível inferior, representa a segunda principal causa de mortes acidentais ou não intencionais em todo o mundo. De acordo com a Organização Mundial da Saúde (OMS), estima-se que 37,3 milhões de quedas graves ocorrem a cada ano e que cerca de 646.000 pessoas morrem todos os anos no mundo devido às quedas ${ }^{(1)}$.

No ambiente hospitalar, as quedas estão associadas não somente ao aumento nas taxas de mortalidade, mas também ao tempo e aos custos de internação, além de contribuir para diminuição na qualidade de vida(2). Estudo realizado no Japão, com 49.059 pacientes hospitalizados durante período de um ano, evidenciou que, nesse intervalo de tempo, ocorreram eventos adversos em $12 \%$ dos casos analisados e $1,7 \%$ destes eram relacionados a quedas ${ }^{(3)}$. Outro estudo, realizado na Austrália, constatou que pacientes com lesão relacionada à queda tiveram, em média, aumento de 4 dias no tempo de internação e de 4.727 dólares nos custos hospitalares ${ }^{(4)}$.

Nesse contexto, a identificação do risco de queda é crucial para a implementação de intervenções preventivas(5) e a Enfermagem pode contribuir no planejamento de ações em conjunto com a equipe multidisciplinar ${ }^{(6,7)}$ através da utilização de ferramentas específicas de sua prática profissional, como o Processo de Enfermagem (PE), baseado nos diagnósticos de Enfermagem da NANDA Internacional (NANDA-I), na classificação dos resultados de Enfermagem (NOC) e na classificação das intervenções de Enfermagem (NIC) ${ }^{(8)}$.

A taxonomia da NANDA-I traz como diagnóstico de Enfermagem o "risco de queda" definido como suscetibilidade aumentada a quedas que pode causar dano físico e comprometer a saúde ${ }^{(9)}$. Ao empregar o diagnóstico de risco, o profissional de Enfermagem pode propor estratégias de promoção da saúde através de programas de prevenção de quedas e sugestões para melhorar a assistência no ambiente hospitalar ${ }^{(8)}$.

Este estudo torna-se relevante pela possibilidade de subsidiar a fundamentação do conhecimento de enfermeiros sobre o risco de queda presente no ambiente hospitalar, além de estimular o uso de plano de cuidados baseado nas terminologias NANDA, NOC e NIC na prática clínica da Enfermagem. Para os pacientes, a identificação precoce da presença de risco de queda refletirá em melhoria na qualidade da assistência recebida, tendo em vista que haverá a implementação das condutas para cada caso específico, demonstrando segurança no cuidado prestado. 
Destaca-se, ainda, que o estudo está alinhado aos princípios da Declaração de Budapeste sobre Hospitais Promotores de Saúde, que afirma ser importante a promoção da saúde nos serviços de atenção terciária e que os usuários devem ser estimulados a ter papel ativo e participativo no cuidado, de acordo com suas potencialidades específicas de saúde. Além disso, as ações sobre segurança do paciente são essenciais para a qualidade de vida do indivíduo hospitalizado(10).

Nessa perspectiva, a cultura de segurança do paciente destaca a importância da proatividade da equipe de Enfermagem na prevenção de quedas a partir de estratégias que possibilitem a avaliação holística do indivíduo, favorecendo um cuidado sistematizado bem como um ambiente seguro. Logo, este estudo teve como objetivo identificar fatores de risco de queda de pacientes hospitalizados e propor atuações de Enfermagem.

\section{MÉTODOS}

Trata-se de estudo descritivo e transversal, com abordagem quantitativa, realizado em um hospital referência da região Norte do estado do Ceará, Brasil. A entidade filantrópica atende cerca de 40 mil pacientes por mês, de mais de 60 municípios da região, com população de, aproximadamente, dois milhões de habitantes, contando com mais de 450 leitos $^{(11)}$.

Realizou-se o presente estudo no período de junho a setembro de 2017, em enfermaria de clínica médica composta por 56 leitos, dentre os quais, 29 são destinados para pacientes clínicos e 27, para traumatológicos. A equipe era composta por seis técnicos de enfermagem e um enfermeiro por plantão, além de médicos e residentes.

Compuseram a população-alvo os pacientes internados na clínica médica no período da coleta de dados. Realizou-se o cálculo amostral com base nos dados do número de internações relativos ao período de janeiro a dezembro de 2016. Neste ano, 513 pacientes foram assistidos na unidade, com média de 42,75 pacientes por mês. Utilizou-se a fórmula para estimativa percentual, com erro amostral de $5 \%$, intervalo de confiança de $95 \%(n=513)$ e proporção de ocorrência do desfecho de 15\%, resultando em 142 pacientes. Entretanto, durante o período de coleta de dados, obteve-se uma amostra superior, representada por 155 pacientes.

Os critérios de inclusão utilizados incluíam: estar internado na clínica médica em estudo, no período mínimo de 24 horas, e ser alfabetizado, para que houvesse compreensão do instrumento utilizado. Já os critérios de exclusão eram: pacientes com indicação de transferência para outro setor do hospital.

Para coleta de dados, utilizou-se uma entrevista norteada por instrumento estruturado e validado que contemplava duas partes: dados clínico-epidemiológicos e diagnóstico de Enfermagem de risco de queda. As coletas foram realizadas por três pesquisadores, devidamente treinados para aplicação do instrumento e avaliação dos riscos no ambiente.

$\mathrm{Na}$ avaliação dos dados clínico-epidemiológicos, coletaram-se informações como sexo, idade, estado civil, nível de escolaridade, procedência, renda, diagnóstico médico e medicamentos. Já o diagnóstico de Enfermagem de risco de queda avaliou a presença dos fatores de risco, conforme a taxonomia da NANDA-I. Para a inferência do diagnóstico de Enfermagem "risco de queda", utilizou-se o referencial de Risner ${ }^{(12)}$, que estabelece as etapas de análise e síntese. Com base nos principais fatores de risco identificados, houve a tabulação e análise das respostas dos pacientes pelos pesquisadores, separadamente, com posterior discussão em conjunto. Então, elaborou-se um plano de cuidados com base nos resultados encontrados por meio da NOC, a qual padroniza o nome e as definições de resultados para uso na prática, educação e pesquisa ${ }^{(13)}$. Para as atuações dos Processos de Enfermagem, utilizou-se a NIC, que propõe diversas atividades que o enfermeiro pode realizar com o indivíduo e a comunidade ${ }^{(14)}$.

Realizou-se a análise estatística no programa SPSS, versão 24, para obtenção de frequências absolutas e percentuais, médias e desvio-padrão. Adotou-se nível de significância de $5 \%$ e intervalo de confiança de $95 \%$. Ao final, após análise e tabulação dos dados, realizou-se a confrontação com a literatura pertinente.

O estudo obteve a aprovação do Comitê de Ética em Pesquisa (CEP) da Universidade Estadual Vale do Acaraú, com o Parecer $n .{ }^{\circ} 2102871$. O estudo atendeu às normas nacionais e internacionais de ética em pesquisa envolvendo seres humanos.

\section{RESULTADOS}

Dos 155 participantes do estudo, 25,16\% ( $n=39)$ eram do sexo feminino e $74,83 \%(n=116)$ do sexo masculino, com idade média de 47,69 anos $( \pm 20,29)$. Quanto à ocupação, $54,83 \%(n=85)$ eram ativos e $45,16 \%(n=70)$ inativos. A maioria dos participantes $(62,58 \% ; n=97)$ era casada, enquanto $27,09 \%(n=42)$ estavam solteiros e $5,16 \%(n=8)$ eram viúvos e divorciados. 
Referente à religião, 84,45\% $(n=134)$ eram católicos, 10,96\% $(n=17)$ evangélicos e 2,58\% $(n=4)$ ateus. O grau de escolaridade predominante foi de 0 a 8 anos de estudo, com $68,38 \%(n=106)$, seguido de 9 a 11 anos de estudo $(27,74 \% ; n=43)$ e $3,87 \%(n=6)$ possuíam mais de 12 anos de estudo. Observou-se que $41,2 \%(n=64)$ dos pacientes tinham no diagnóstico causas clínicas e $58,8 \%(n=91)$ traumatológicas. A média do tempo de internação foi de 8,52 $( \pm 8,11)$ dias.

Os principais fatores relacionados ao diagnóstico de risco de queda nos pacientes da clínica médica possuem relação com características do paciente, envolvendo problemas fisiológicos e o uso de medicamentos, além de estarem inseridos em ambiente de risco. Houve destaque para idade acima de 65 anos, uso de dispositivos auxiliares, história de queda, anemia, insônia, pós-operatório, dificuldade para deambular, força diminuída nas extremidades, equilíbrio prejudicado, desconhecimento do ambiente, insuficiência de material antiderrapante nos banheiros e agentes farmacológicos (Tabela I).

Tabela I - Distribuição dos fatores de risco para diagnóstico de enfermagem "risco de queda" em pacientes avaliados na clínica médica de instituição hospitalar (n=155). Sobral, Ceará, Brasil, 2017.

\begin{tabular}{|c|c|c|}
\hline Fatores de risco em adultos & $\mathbf{n}$ & $\%$ \\
\hline Prótese de membro inferior & 2 & 1,29 \\
\hline Uso de cadeira de rodas & 11 & 7,09 \\
\hline Idade acima de 65 anos & 25 & 16,12 \\
\hline Uso de dispositivos auxiliares & 43 & 27,74 \\
\hline História de queda & 43 & 27,74 \\
\hline \multicolumn{3}{|l|}{ Cognitivos } \\
\hline Alterações na função cognitiva & 6 & 3,87 \\
\hline \multicolumn{3}{|l|}{ Fisiológicos } \\
\hline Doença vascular & 4 & 2,58 \\
\hline Alteração no nível de glicose no sangue & 5 & 3,22 \\
\hline Artrite & 5 & 3,22 \\
\hline Déficits proprioceptivos & 6 & 3,87 \\
\hline Incontinência & 6 & 3,87 \\
\hline Neuropatia & 6 & 3,87 \\
\hline Urgência urinária & 6 & 3,87 \\
\hline Diarreia & 8 & 5,16 \\
\hline Dificuldades auditivas & 8 & 5,16 \\
\hline Neoplasias & 8 & 5,16 \\
\hline Dificuldades visuais & 20 & 12,9 \\
\hline Hipotensão ortostática & 20 & 12,9 \\
\hline Condição que afeta os pés & 22 & 14,19 \\
\hline Ausência de sono & 28 & 18,06 \\
\hline Anemia & 30 & 19,35 \\
\hline Pós-operatório & 44 & 28,38 \\
\hline Força diminuída nas extremidades & 52 & 33,54 \\
\hline Equilíbrio prejudicado & 74 & 47,74 \\
\hline Mobilidade física prejudicada & 79 & 50,96 \\
\hline Dificuldade na marcha & 88 & 56,77 \\
\hline \multicolumn{3}{|l|}{ Ambientais } \\
\hline Uso de tapetes soltos & - & - \\
\hline Iluminação insuficiente & 2 & 1,29 \\
\hline Uso de imobilizadores & 2 & 1,29 \\
\hline Ambiente desorganizado & 5 & 3,22 \\
\hline Exposição à condição insegura & 5 & 3,22 \\
\hline Cenário pouco conhecido & 39 & 25,16 \\
\hline Material antiderrapante insuficiente no banheiro & 46 & 26,67 \\
\hline \multicolumn{3}{|l|}{ Agentes farmacológicos } \\
\hline Consumo de álcool & 18 & 11,61 \\
\hline Agente farmacológico & 39 & 25,16 \\
\hline
\end{tabular}


Após a identificação dos fatores para o diagnóstico risco de queda, elaborou-se um plano de cuidados considerando os resultados almejados por NOC e as intervenções descritas pela NIC, conforme exposto no Quadro I.

Quadro I - Plano de cuidados com base nos resultados esperados descritos pela NOC (classificação dos resultados de Enfermagem) e as intervenções descritas pela NIC (classificação das intervenções de Enfermagem) em pacientes com diagnóstico de Enfermagem de risco de queda avaliados na clínica médica de instituição hospitalar ( $\mathrm{n}=155)$. Sobral, Ceará, Brasil, 2017.

\begin{tabular}{|c|c|c|}
\hline $\begin{array}{l}\text { Diagnóstico de } \\
\text { enfermagem } \\
\text { (NANDA) }\end{array}$ & $\begin{array}{l}\text { Resultados esperados } \\
\text { (NOC) }\end{array}$ & $\begin{array}{l}\text { Intervenções de enfermagem } \\
\text { (NIC) }\end{array}$ \\
\hline $\begin{array}{l}\text { Risco de queda } \\
(00155)\end{array}$ & $\begin{array}{l}\text { (0200) Locomoção: caminhar } \\
\text { (0201) Locomoção: cadeira de rodas } \\
\text { (0202) Equilíbrio } \\
\text { (2300) Nível de glicemia } \\
\text { (0500) Continência intestinal } \\
\text { (0501) Eliminação intestinal } \\
\text { (0212) Movimento coordenado } \\
\text { (1909) Comportamento de prevenção de queda } \\
\text { (0007) Nível de fadiga } \\
\text { (0602) Hidratação } \\
\text { (1809) Conhecimento: Segurança pessoal } \\
\text { (1828) Conhecimento: Prevenção de quedas } \\
\text { (2301) Resposta a medicação } \\
\text { (0208) Mobilidade } \\
\text { (0917) Estado neurológico: Periférico } \\
\text { (0113) Envelhecimento físico } \\
\text { (2004) Aptidão física } \\
\text { (1902) Controle de riscos } \\
\text { (0310) Autocuidado: uso do banheiro } \\
\text { (0004) Sono } \\
\text { (0210) Desempenho na transferência } \\
\text { (0502) Continência urinária }\end{array}$ & \begin{tabular}{|l} 
(0221) Terapia com exercício: deambulação \\
(0846) Posicionamento: cadeira de rodas \\
(0222) Terapia com exercício: equilíbrio \\
(2120) Controle da hiperglicemia \\
(2130) Controle da hipoglicemia \\
(0410) Cuidados na incontinência intestinal \\
(0430) Controle de intestinal \\
$(0201)$ Promoção do exercício: treino para fortalecimento \\
(6490) Prevenção contra queda \\
(0224) Terapia com exercício: mobilidade articular \\
(4120) Controle hídrico \\
(6486) Controle do ambiente segurança \\
(6490) Prevenção contra quedas \\
(2380) Controle de medicamentos \\
$(0202)$ Promoção do exercício: alongamento \\
(1400) Controle da dor; \\
$(2660)$ Controle da sensibilidade periférica \\
(1800) Assistência no autocuidado \\
$(0226)$ Terapia com exercício: controle muscular \\
(6610) Identificação de risco \\
$(1804)$ Assistência no autocuidado: uso de vaso sanitário \\
(1850) Melhora do sono \\
(1806) Assistência no autocuidado: transferências \\
(0610) Cuidados na incontinência urinária
\end{tabular} \\
\hline
\end{tabular}

\section{DISCUSSÃO}

O perfil dos participantes do presente estudo foi de: homens, com média de idade de 47 anos, ativos, casados, católicos e com até 8 anos de estudo. Corroborando esses dados, outros estudos, realizados no Sul do Brasil(15) e em Minas Gerais ${ }^{(16)}$, identificaram perfil semelhante de pacientes hospitalizados. No atual estudo, os achados se justificam pelo grande número de pacientes vítimas de trauma e sua relação intrínseca com homens em idade ativa.

Em relação aos fatores de risco, todos os pacientes da atual pesquisa apresentaram pelo menos um risco associado à queda durante o período de hospitalização. Os principais fatores de risco foram relacionados a condições fisiológicas, uso de agentes farmacológicos, dificuldade na marcha, mobilidade prejudicada e equilíbrio prejudicado. Também esteve presente o risco ambiental, representado por material antiderrapante insuficiente no banheiro e cenário pouco conhecido.

Outras pesquisas também identificaram fatores de risco semelhantes para inferir o risco de queda, como condições ambientais, alterações neurológicas, mobilidade prejudicada, efeitos adversos de medicação, extremos de idade e alterações fisiológicas ${ }^{(15,16)}$.

A partir do conhecimento dos principais fatores de risco, o enfermeiro, como agente promotor da saúde, tem subsídios para criar um ambiente seguro e estabelecer estratégias que contribuam para redução da ocorrência de quedas. As atuações para "prevenção contra quedas", dispostas na NIC, são amplas, representadas por 65 atividades que podem ser desempenhadas em diferentes cenários de saúde. Essas atividades são agrupadas em dimensões cognitivas, ambientais, de equilíbrio, transferência, uso de dispositivos de saúde, educação em saúde, estruturas hospitalares e intrahospitalares, e sistemas de alarme que podem ser direcionadas conforme as principais necessidades do paciente ${ }^{(8,14)}$. 
É pertinente que as atuações para "prevenção contra quedas" sejam direcionadas para o contexto dos indivíduos e, ao promover saúde no ambiente hospitalar com o intuito de reduzir as quedas, é preciso utilizar protocolos específicos, um plano de cuidados direcionado e educação em saúde. A implementação de intervenções, com enfoque nos fatores de risco existentes, possibilita a redução dos indicadores e suas consequências ${ }^{(17)}$.

No presente estudo, foram sugeridas intervenções da NIC com base nos resultados de enfermagem da NOC. Um dos fatores de risco predominantes nesse estudo foi "idade acima de 65 anos" e, de acordo com o National Council on Aging, a cada 11 segundos, um idoso é admitido na emergência devido à queda e, a cada 19 minutos, um idoso morre por complicações após queda(18).

Embora o risco de queda se torne maior à medida que o indivíduo envelhece, esse evento adverso é causado por complexa interação de fatores de risco biológicos, comportamentais, ambientais e socioeconômicos. A idade por si só não deve determinar intervenções. Entretanto, é pertinente a avaliação cuidadosa dos riscos e o fornecimento de intervenções apropriadas, incluindo tratamento de condições clínicas, revisão de medicamentos, ensino de exercícios e educação sobre segurança em prevenção de quedas, pois podem contribuir para um envelhecimento mais saudável(19).

Um dos pilares da promoção da saúde é o incentivo à prática de atividade física para manutenção de estilo de vida mais saudável. Assim, os profissionais de saúde podem orientar exercícios de equilíbrio e força durante as atividades diárias com os pacientes e familiares ${ }^{(20)}$.

Em casos em que os fatores de risco são: "diarreia", "incontinência" e "urgência urinária", a Enfermagem deve prestar assistência com cuidados na incontinência intestinal, controle intestinal, controle hídrico e cuidados na continência urinária. Estudo realizado na China analisou eventos adversos, registrados em prontuários de 818 leitos, e revelou risco de queda consideravelmente maior em pacientes com eliminações prejudicadas ${ }^{(21)}$. Nesses casos, é importante a revisão do uso de medicamentos, como diuréticos e laxantes, além do auxílio ao conforto contínuo, com a troca frequente de fraldas, caso necessário, ou rotina com horários regulares para levá-lo ao banheiro ${ }^{(22)}$.

No fator de risco "insônia", as intervenções devem possibilitar maior qualidade de sono ao paciente. Estudo de coorte retrospectivo canadense evidenciou que as quedas ocorrem, frequentemente, à noite, e não são vistas pelos profissionais de saúde, sendo associadas à insônia, alterações cognitivas nos pacientes e mudança dos plantonistas da equipe enfermagem ${ }^{(23)}$.

Muitos dos fatores associados à queda são modificáveis e evitáveis, logo, atitudes voltadas à educação em saúde e prevenção podem interferir positivamente e reduzir as chances de queda ${ }^{(24)}$.

Sabe-se que a prevenção de quedas em pacientes hospitalizados vem se tornando objeto de investigação em âmbito global, uma vez que traz repercussões diretas para a segurança do paciente e para a qualidade da assistência. A implementação de ações para prevenção de quedas com a utilização de instrumentos de avaliação de riscos e sensibilização/orientação de pacientes, familiares e trabalho em equipe diminuem a ocorrência de queda(25).

Sugere-se, então, que programas de promoção da saúde com enfoque na redução de quedas não sejam implementados apenas nas enfermarias, com pacientes e familiares, mas também ocorram nos corredores, nas salas de espera e em outros ambientes da instituição hospitalar, com o objetivo de alcançar um maior número de pessoas e, assim, sensibilizar sobre os riscos de queda existentes no hospital.

As principais limitações do atual estudo foram o fato de apenas um setor do serviço hospitalar ter sido incluído no levantamento dos dados. Dessa forma, não foi possível comparar as particularidades de cada setor e sua associação com o risco de queda. Outra limitação foi o fato das atuações dos processos de Enfermagem não terem sido implementadas com os pacientes, o que possibilitaria a avaliação da efetividade e aplicabilidade na prática clínica.

Reconhecendo-se os riscos inerentes a cada paciente idoso e que é possível a implementação de estratégias para a prevenção de quedas, como a educação em saúde, com objetivo de capacitar esses indivíduos para atuarem no autogerenciamento dos riscos, aumentando, assim, a adesão aos cuidados orientados e, consequentemente, reduzindo esse evento adverso. Diante da necessidade de fornecer assistência de qualidade aos pacientes hospitalizados, a avaliação dos riscos de queda e a construção de estratégias de prevenção são essenciais. A elaboração do plano de cuidados possibilita a tomada de decisão clínica do enfermeiro durante a assistência, o que favorece um cuidado seguro e livre de danos.

\section{CONCLUSÃO}

A identificação do diagnóstico de Enfermagem "risco de queda" permitiu evidenciar o predomínio de fatores de risco, como idade acima de 65 anos, uso de dispositivos auxiliares, história de queda, anemia, insônia, pós- 
operatório, dificuldade para deambular, força diminuída nas extremidades, equilíbrio prejudicado, desconhecimento do ambiente, insuficiência de material antiderrapante nos banheiros e presença de agentes farmacológicos em pacientes hospitalizados na clínica médica.

As principais atuações a serem implementadas para prevenção da ocorrência de queda são: promoção do exercício, controle da dor, prevenção contra queda, controle do ambiente, assistência no autocuidado e educação em saúde.

\section{CONFLITOS DE INTERESSE}

Autores declaram que não há conflitos de interesses.

\section{FONTE DE FINANCIAMENTO}

Hospital Santa Casa de Misericórdia de Sobral, pelo edital DEPE n. ${ }^{\circ}$ 02/2017.

\section{CONTRIBUIÇÕES}

Maria Aline Moreira Ximenes e Jefferson Ribeiro Aguiar, contribuíram com a elaboração e delineamento do estudo; aquisição, análise e interpretação de dados; e a redação e/ou revisão do manuscrito. Ismael Brioso Bastos, Liliane Vieira de Sousa e Joselany Áfio Caetano contribuíram com a redação e/ou revisão do manuscrito. Lívia Moreira Barros contribuiu com a aquisição, análise e interpretação de dados; e a redação e/ou revisão do manuscrito.

\section{REFERÊNCIAS}

1. World Health Organization. Falls [Internet]. Geneva: WHO; 2018 [access on 2019 Sept 6]. Available from: http://www.who.int/mediacentre/factsheets/fs344/en/

2. Souza AB, Maestri RN, Röhsig V, Lorenzini E, Alves BM, Oliveira D, et al. In-hospital falls in a large hospital in the south of Brazil: A 6-year retrospective study. Appl Nurs Res [Internet]. 2019 [access on 2019 Nov 5];48:8187. doi: 10.1016/j.apnr.2019.05.017

3. Kazuyoshi K, Shiro I, Yuko I, Yusuke S, Kei A, Yoshihiro N, et al. Incidence and characteristics of accidental falls in hospitalizations. Nagoya J Med Sci [Internet]. 2017 [access on 2018 Nov 4];79(3):291-98. doi: 10.18999/nagjms.79.3.291

4. Morello RT, Barker AL, Watts JJ, Haines T, Zavarsek SS, Hill KD, et al. The extra resource burden of inhospital falls: a cost of falls study. Med J Aust [Internet]. 2015 [access on 2018 Nov 4];203(9):367e. doi: 10.5694/mja15.00296

5. Lusardi MM, Fritz S, Middleton A, Allison L, Wingood M, Phillips E, et al. Determining risk of falls in community dwelling older adults: a systematic review and meta-analysis using posttest probability. J Geriatr Phys Ther [Internet]. 2017 [access on 2018 Nov 6];40(1):1-36. Available from: https://www.ncbi.nlm.nih.gov/ pubmed/27537070

6. Toye C, Kitchen S, Hill A, Edwards D, Sin M, Maher S. Piloting staff education in Australia to reduce falls in older hospital patients experiencing delirium. Nurs Health Sci [Internet]. 2017 [access on 2018 Nov 5];19(1):51-8. doi: 10.1111/nhs.12300

7. Pasa TS, Magnago TSBS, Urbanetto JS, Baratto MAM, Morais BX, Carollo JB. Risk assessment and incidence of falls in adult hospitalized patients. Rev Latinoam Enferm [Internet]. 2017 [access on $2018 \mathrm{Nov}$ 5];25:e2862. doi: 10.1590/1518-8345.1551.2862

8. Hernández-Herrera D, Aguilera-Elizarraraz N, Vega-Argote M, González-Quirarte N, Castañeda-Hidalgo H, Isasi-Hernández L. Aplicación de las actividades de la intervención de enfermería Prevención de caídas en adultos hospitalizados. Enferm Univ [Internet]. 2017 [access on 2018 Nov 5];14(2):118-23. doi: 10.1016/j. reu.2017.02.007

9. NANDA International. Diagnósticos de enfermagem da NANDA: definições e classificação 2015 - 2017. Porto Alegre: Artmed; 2015. 
10. Pereira FGF, Matias EO, Caetano JA, Lima FET. Segurança do paciente e promoção da saúde: uma reflexão emergente. Rev Baiana Enferm [Internet]. 2015 [access em 2019 Out 17];29(3):271-77. doi: 10.18471/rbe. v29i3.12205

11. Thiago E. Histórico [Internet]. Sobral: Santa Casa de Misericórdia de Sobral; 2016 [access on 2018 Jan 11]. Available from: http://stacasa.com.br/site/hitorico/

12. Risner PB. Nursing diagnosis: diagnostic sistements. In: Christensen PJ, Kenney JW. Nursing process: application of conceptual models. 3rd ed. St. Louis: Mosby; 1990. Cap. 7, p. 132-57.

13. Moorhead S, Johnson M, Maas M, Swanson E. Classificação dos Resultados de Enfermagem: mensuração dos resultados em saúde. 5rd ed. Rio de Janeiro: Elsevier; 2016.

14. Bulechek GM, Butcher HK, Dochterman JM, Wagner CM. Classificação das intervenções de enfermagem (NIC). Grd ed. Rio de Janeiro: Elsevier; 2016.

15. Luzia MF, Almeida MA, Lucena AF. Mapeamento de cuidados de enfermagem para pacientes com risco de quedas na Nursing Interventions Classification. Rev Esc Enferm USP [Internet]. 2014 [access on 2019 Sept 5];48(4):632-40. Available from: http://www.scielo.br/scielo.php?script=sci_ arttext\&pid=S008062342014000400632\&lng=en\&nrm=isso

16. Mata L, Azevedo C, Policarpo A, Moraes J. Fatores associados ao risco de queda em adultos no pósoperatório: estudo transversal. Rev Latinoam Enferm [Internet]. 2017 [acesso em 2019 Set 5];25:e2904. Disponível em: http://www.revistas.usp.br/rlae/article/view/134960

17. Araújo MF, Caldevilla NN, Maciel C, Malheiro F, Rodríguez-Borrego MA, López-Soto PJ. Record of the circumstances of falls in the community: perspective in the Iberian Peninsula. Rev Latinoam Enferm [Internet]. 2018 [access on 2019 Out 17];26:e2977. doi: 10.1590/1518-8345.2373.2977

18. National Council on Aging. Falls prevention: keeping older adults safe and active [Internet]. Arlington: NCA; 2018 [access on 2019 Out 17]. Available from: https://www.ncoa.org/healthy-aging/falls-prevention/

19. Bolding DJ, Corman E. Falls in the Geriatric Patient. Clin Geriatr Med [Internet]. 2019 [access on 2019 Out 17];35(1):115-26. doi: 10.1016/j.cger.2018.08.010

20. Kulinski K, DiCocco C, Skowronski S, Sprowls P. Advancing community-based falls prevention programs for older adults-the work of the administration for community living/administration on aging. Front Public Health [Internet]. 2017 [access on 2019 Out 17];5:4. doi: 10.3389/fpubh.2017.00004

21. Hou WH, Kang CM, Ho MH, Kuo JM, Chen HL, Chang WY. Evaluation of an inpatient fall risk screening tool to identify the most critical fall risk factors in inpatients. J Clin Nurs [Internet]. 2017 [access on 2018 Nov 6];26(56):698-706. doi: 10.1111/jocn.13510

22. Ministério da Saúde (BR). Protocolo para prevenção de quedas [Internet]. Brasília: Ministério da Saúde; 2013 [access on 2018 Nov 6]. Disponível em: https://www20.anvisa.gov.br/segurancadopaciente/index.php/ publicacoes/item/prevencao-de-quedas

23. Trumble D, Meier MA, Doody M, Wang X, Bagshaw SM. Incidence, correlates and outcomes associated with falls in the intensive care unit: a retrospective cohort study. Crit Care Resusc [Internet]. 2017 [access on 2018 Nov 7];19(4):290-5. Available from: https://www.ncbi.nlm.nih.gov/pubmed/29202254

24. Lima AP, Lini EV, Dellani MP, Portella MR, Doring M. Prevalência e fatores associados às quedas em idosos de Estação-RS: estudo transversal de base populacional. Cad Saúde Colet [Internet]. 2017 [acesso em 2018 Nov 7];25(4):436-42. doi: 10.1590/1414-462×201700040271

25. Luzia MF, Argenta C, Almeida MA, Lucena AF. Definições conceituais dos indicadores do resultado de enfermagem "Conhecimento: Prevenção de quedas". Rev Bras Enferm [Internet]. 2018 [acesso em 2018 Nov 06];71(2):461-70. doi: 10.1590/0034-7167-2016-0686 


\section{Endereço para correspondência:}

Maria Aline Moreira Ximenes

Universidade Estadual Vale do Acaraú - UVA

Av. da Universidade, 850

Bairro: Campus da Betânia

CEP: 62.040-370 - Sobral - CE - Brasil

E-mail: aline.ximenes11@hotmail.com

Como citar: Ximenes MAM, Aguiar JR, Bastos IB, Sousa LV, Caetano JA, Barros LM. Risco de queda de pacientes hospitalizados: fatores de risco e atuações de enfermagem. Rev Bras Promoç Saúde. 2019;32:9003. 